\title{
Estimate of the incoherent-scattering contribution to lidar backscatter from clouds
}

\author{
David A. de Wolf, Herman W. J. Russchenberg, and Leo P. Ligthart
}

\begin{abstract}
Lidar backscatter from clouds in the Delft University of Technology experiment is complicated by the fact that the transmitter has a narrow beam width, whereas the receiver has a much wider one. The issue here is whether reception of light scattered incoherently by cloud particles can contribute appreciably to the received power. The incoherent contribution can come from within as well as from outside the transmitter beam but in any case is due to at least two scattering processes in the cloud that are not included in the coherent forward scatter that leads to the usual exponentially attenuated contribution from single-particle backscatter. It is conceivable that a sizable fraction of the total received power within the receiver beam width is due to such incoherent-scattering processes. The ratio of this contribution to the direct (but attenuated) reflection from a single particle is estimated here by means of a distorted-Born approximation to the wave equation (with an incident $\mathrm{cw}$ monochromatic wave) and by comparison of the magnitude of the doubly scattered to that of the singly scattered flux. The same expressions are also obtained from a radiative-transfer formalism. The ratio underestimates incoherent multiple scattering when it is not small. Corrections that are due to changes in polarization are noted. (C) 1999 Optical Society of America
\end{abstract}

OCIS codes: $\quad 290.1350,010.1310,290.1310,260.2110,290.1090,290.4210,290.7050$.

\section{Introduction}

In this research we treat the analysis of backscattered reflections from a small lidar range cell that contains liquid cloud particles well into the cloud. If it is accepted that electromagnetic power scattered at all but very small angles out of the beam, both to and from the range cell, cannot return to the receiver, then one can account for the effect of the particles outside the immediate environment of the line of sight by means of an exponential attenuation factor in the backscatter cross section of the range cell.1,2 This contribution is considered coherent because multiple scattering from particle to particle at (infinitesimally) small angles does not change the phase of

When this research was performed, all the authors were with the International Research Centre for TelecommunicationsTransmission and Radar, Faculty of Information Technology and Systems, Delft University of Technology, 2628 CD Delft, The Netherlands. D. A. de Wolf is now with the Bradley Department of Electrical Engineering, Center for Stochastic Processes in Science and Engineering, Virginia Polytechnic Institute and State University, 340 Whittemore Hall, Blacksburg, Virginia 24061-0111. The email address for D. A. de Wolf is dadewolf@vt.edu.

Received 20 January 1998; revised manuscript received 15 October 1998.

0003-6935/99/030585-09\$15.00/0

(C) 1999 Optical Society of America the electric field from the free-space value. It is a dominant contribution for particles large compared with the wavelength because the scattering pattern is highly peaked at zero scattering angle. This contribution resembles that which produces an effective refractive index for the medium ${ }^{3}$ but differs because the interparticle distance is large compared with the wavelength. 4 At infrared and optical frequencies, most cloud particles are also no longer large compared with the wavelength, and electromagnetic scattering then is closer to being isotropic. Hence the question arises to what extent electromagnetic power scattered out of the line of sight can reenter it and contribute to the backscatter cross section. This possibility is augmented in the clouds and radiation experiments in The Netherlands ${ }^{5}$ by the fact that the receiving beam width is much wider than that of the transmitter so the receiver may process direct contributions from off-axis particles. These contributions are incoherent because the large-angle scatterings produce sizable phase differences in the field contributions at the receiver, with a sum that tends to be incoherent. The optical thickness of the region traversed by the beam plays a significant role in the importance of the incoherent contribution. These contributions modify the coherent contribution by adding incoherent terms to the total received field, which may or may not yield significant contributions 
to the irradiance, depending on the optical thickness of the traversed region.

When the incoherent contributions dominate, it is customary to invoke radiative-transfer theory and to label them multiple-scattering contributions. The connection to Maxwell theory was discussed recently by Kravtsov and Apresyan. ${ }^{6}$ A recent comparison with the single-scattering contribution was made by Mannoni et al..$^{7}$ for Chebyshev particles, by Nicolas et $a l .{ }^{8}$ for ice crystals in cirrus clouds, by Xu et al. ${ }^{9}$ for various cloud-particle distributions, and by Flesia and Starkov ${ }^{10}$ for clear-air versus ice-crystal contributions in a cloud. One of the earliest similar calculations was made by Bruscaglioni, ${ }^{11}$ who compared a double-scattering calculation with a singlescattering one. A difference from the present study is that his does not include exponential attenuation factors in the volume-scattering integral. These factors are needed here because the present calculations are based on a cw monochromatic incident spherical wave. As explained below for assumption II, inclusion of the exponential factor allows us to estimate when multiple incoherent scattering must be considered without incurring the difficulties of the usual pulsed-beam approach.

A recent issue of Applied Physics B contains several related papers that deal with lidar backscatter from a laboratory aerosol. Bissonnette ${ }^{12}$ works out a radiative-transfer model in a paraxial-diffusion approximation, whereas Bruscaglioni et al. ${ }^{13}$ perform Monte Carlo calculations on the same aerosol. Flesia and Schwendimann ${ }^{14}$ extend Mie theory analytically, and Starkov et al..$^{15}$ compare the transporttheoretical approach with a stochastic model by using Monte Carlo techniques. Winker and Poole ${ }^{16}$ also perform a Monte Carlo backscatter calculation, whereas Zege et al. ${ }^{17}$ present a complete analytical and a simplified semianalytical solution to the radiative-transfer equations. Finally, these efforts are compared in a summary paper by Bissonnette $e t$ $a l .{ }^{18}$ The radiative-transfer methods generally require some parameters to be adjusted to measured quantities. All these simulations are based on an incident pulsed beam. A different approach is taken in the present paper.

\section{Analysis}

This study is based on a renormalized version of the multiple-scattering equation ${ }^{19}$ for the propagation of monochromatic electromagnetic waves in particulate media:

$$
\begin{aligned}
\mathbf{E}= & \mathbf{E}_{0}+\sum_{\alpha} \overleftrightarrow{\mathbf{t}}_{\alpha} \cdot \mathbf{E}_{0}+\sum_{\alpha} \sum_{\beta \neq \alpha} \overleftrightarrow{\mathbf{t}}_{\alpha} \cdot \overleftrightarrow{\mathbf{t}}_{\beta} \cdot \mathbf{E}_{0} \\
& +\sum_{\alpha} \sum_{\beta \neq \alpha} \sum_{\gamma \neq \beta} \overleftrightarrow{\mathbf{t}}_{\alpha} \cdot \overleftrightarrow{\mathbf{t}}_{\beta} \cdot \overleftrightarrow{\mathbf{t}}_{\gamma} \cdot \mathbf{E}_{0}+\ldots
\end{aligned}
$$

Equation (1) is a symbolic operator form of the actual equations. $\mathbf{E}_{0}(\mathbf{r})$ is the field at $\mathbf{r}$ that is due to a monochromatic plane wave emitted by the source in the absence of a medium. The dyadic operator $\overleftrightarrow{\mathbf{t}}_{\alpha}$ represents the sum of all repeated scatterings that occur inside particle $\alpha$. Several assumptions are made for tractability of the ensuing analysis (which is considerably simpler than that for pulsed incident radiation):

Assumption I. The cloud is a uniform monodisperse distribution of spherical particles between altitudes $z=R$ and $z=R+L$. The drop distribution certainly is not monodisperse, but the calculations become unnecessarily cumbersome if a polydisperse distribution is assumed.

Assumption II. The reflection from a single particle at $z=R$ as the result of an incident monochromatic spherical wave is a reasonable measure of the desired effect. When pulses rather than monochromatic waves are used, the reflection will come from a region of essentially a half-pulse width in height (if multiple scattering is ignored). Multiply scattered power flux will take longer times to return and thus enter into other range cells. By the same token, the singly scattered power flux in the desired range cell will be contaminated by multiple scattering from regions that contribute to lower range cells. The simplified monochromatic cw calculation that we propose below is a reasonable measure of the degree of mixing of multiple scattering to (attenuated) single scattering. That is to say, if the multiple-scattering contribution is negligible here, then it certainly is negligible in the much more difficult to calculate case of reflections from pulsed signals.

Assumption III. Details of antenna-beam patterns and of Mie-scattering patterns will be simplified at various places in the calculation.

Assumption IV. The incoherent scattering contribution will be estimated from the lowest-order multiple-scattering contribution that excludes previously summed coherent contributions: the secondorder distorted-Born term.

Assumption $V$. The particle to be considered is most distant from the lidar. The cloud is positioned at $1-1.5-\mathrm{km}$ altitude (the crucial geometrical parameter is the ratio of cloud thickness to altitude), and the lidar is either on the ground or on a satellite.

The actual form of the third term of Eq. (1) can be shown to be

$$
\begin{aligned}
\mathbf{E}_{\mathrm{sc}}^{(2)}(\mathbf{r})= & k^{4} \sum_{\alpha} \sum_{\beta \neq \alpha} \overleftrightarrow{\mathbf{g}}_{0}\left(\mathbf{r}-\mathbf{r}_{\alpha}\right) \cdot \overleftrightarrow{\mathbf{f}}_{\alpha}\left(\hat{k}_{f \alpha}, \hat{k}_{\alpha \beta}\right) \\
& \cdot \overleftrightarrow{\mathbf{g}}_{0}\left(\mathbf{r}_{\alpha}-\mathbf{r}_{\beta}\right) \cdot \overleftrightarrow{\mathbf{f}}_{\beta}\left(\hat{k}_{\alpha \beta}, \hat{k}_{\beta 0}\right) \cdot \mathbf{E}_{0}(\mathbf{r})
\end{aligned}
$$

Here $k$ is the free-space wave number and $\overleftrightarrow{\mathbf{g}}_{0}\left(\mathbf{r}-\mathbf{r}_{\alpha}\right)$ $\approx\left(\overleftrightarrow{\mathbf{1}}-\hat{k}_{f \alpha} \hat{k}_{f \alpha}\right) g_{0}\left(\mathbf{r}-\mathbf{r}_{\alpha}\right)$, where the scalar part is the free-space Green's function for propagation from $\mathbf{r}_{\alpha}$ to $\mathbf{r}$ (the dyadic part ensures that the electric field is perpendicular to the direction of propagation). This is a high-frequency approximation that assumes, reasonably for cloud particles, that each particle is in the far field of each other particle. The unit vector $\hat{k}_{21}$ represent a direction from $\mathbf{r}_{1}$ to $\mathbf{r}_{2}$; thus $\hat{k}_{f \alpha}$ represents the direction from $\mathbf{r}_{\alpha}$ to $\mathbf{r}$, etc. Likewise, higher- 


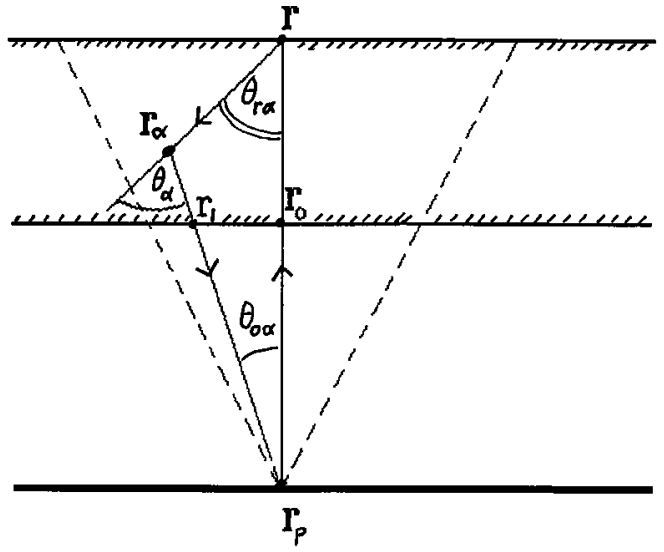

Fig. 1. Sketch of geometry with one intermediate off-axis scattering.

order terms represent more intermediate scatterings from particle to other particle. The scattering amplitude $\overleftrightarrow{\mathbf{f}}_{\alpha}\left(\hat{k}_{f \alpha}, \hat{k}_{\alpha \beta}\right)$ is diagonal for a spherical particle, and it represents the change in magnitude and direction (by angle $\theta$ ) of an incident plane-wave timeharmonic electric field immediately on exit from the particle. The magnitudes of the three coefficients are only functions of the scattering angle $\theta$, and they are given by Mie theory. All the dyadics in Eq. (2) can be considered $2 \times 2$ tensors $^{1}$ with respect to two orthogonal polarizations of the incident field $\mathbf{E}_{0}$, so the two components of $\overleftrightarrow{\mathbf{f}}_{\alpha}$ are $f_{\mathrm{Mie}}$ and $f_{\mathrm{Mie}} \cos \theta$, where $f_{\text {Mie }}(\theta, D)$ is the Mie-scattering coefficient for angle $\theta$ from a spherical particle with diameter $D$.

It is well understood ${ }^{20}$ that a partial summation of all the coherently acting terms in Eq. (1) (i.e., terms for which scattering occurs at vanishingly small angles) leads to

$$
\begin{aligned}
& \mathbf{E}=\mathbf{E}_{0} \exp \left(-\gamma\left|\mathbf{r}-\mathbf{r}_{0}\right|\right), \\
& \gamma=(2 \pi / k) \int_{\mathbf{r}_{0}}^{\mathbf{r}} \mathrm{d} z \int_{0}^{\infty} \mathrm{d} D n_{4}(z, D) \operatorname{Im}\left[f_{\text {Mie }}(0, D)\right] .
\end{aligned}
$$

Here $k=\omega \sqrt{\mu_{0} \varepsilon_{0}}=\omega / c, \gamma$ is the attenuation coefficient in nepers per meter, and $n_{4}(z, D)$ is the particlediameter distribution density in meters to the minusfourth power. The inverse, $1 / \gamma$, is the equivalent of a mean free path through a turbid medium. The exponent $\gamma\left|\mathbf{r}-\mathbf{r}_{0}\right|$ is intended to be shorthand notation for (possibly complex) attenuation in the cloud part of the path; see Fig. 1. We now make the distorted-Born approximations ${ }^{21}$ :

$$
\begin{gathered}
g_{0}\left(\mathbf{r}-\mathbf{r}_{\alpha}\right) \rightarrow g\left(\mathbf{r}-\mathbf{r}_{\alpha}\right) \equiv \exp \left(-\gamma\left|\mathbf{r}-\mathbf{r}_{\alpha}\right|\right) g_{0}\left(\mathbf{r}-\mathbf{r}_{\alpha}\right), \\
g_{0}\left(\mathbf{r}_{\alpha}-\mathbf{r}_{0}\right) \rightarrow g\left(\mathbf{r}_{\alpha}-\mathbf{r}_{0}\right) \equiv \exp \left(-\gamma\left|\mathbf{r}_{\alpha}-\mathbf{r}_{0}\right|\right) g_{0}\left(\mathbf{r}_{\alpha}-\mathbf{r}_{0}\right),
\end{gathered}
$$

with nonzero $\gamma$ only inside the medium. These approximations seems to hold well in Eqs. (1) and (2) as long as particle densities are not so high that the far-field approximations in these equations are violated. Use of $g$ instead of $g_{0}$ amounts to a reordering of the terms in Eq. (1) with partial summations of the coherent contributions. The renormalized second term of Eq. (1) — which is the first term for the backscatter contribution at $\mathbf{r}_{p}$ from a particle with diameter $D$ at $\mathbf{r}$, as shown in Fig. 1 -will be

$$
\mathbf{E}_{\mathrm{bs}}^{(1)}=g\left(\mathbf{r}-\mathbf{r}_{p}\right) f\left(-\hat{k}_{0}, \hat{k}_{0}\right) g\left(\mathbf{r}_{p}-\mathbf{r}\right) \mathbf{E}_{0},
$$

and the associated power will be

$$
\begin{aligned}
P_{\mathrm{bs}}^{(1)} & =\frac{1}{(4 \pi R)^{4}}\left|f\left(-\hat{k}_{0}, \hat{k}_{0}\right)\right|^{2} \exp (-4 \gamma \Delta R), \\
\Delta R & \equiv\left|\mathbf{r}-\mathbf{r}_{0}\right|, \quad \hat{k}_{0}=k \hat{z}
\end{aligned}
$$

or, in a slightly altered notation (to indicate dependence on particle diameter),

$$
P_{\mathrm{bs}}^{(1)}=\frac{1}{(4 \pi R)^{4}}\left|f_{\mathrm{Mie}}(\pi, D)\right|^{2} \exp (-4 \gamma \Delta R) .
$$

The first term of the incoherent contribution [third term of Eq. (1)] will be

$$
\begin{aligned}
\mathbf{E}_{\mathrm{bs}}^{(2)}= & \sum_{\alpha} g\left(\mathbf{r}-\mathbf{r}_{\alpha}\right) f\left(\hat{k}_{\alpha 0}, \hat{k}_{r \alpha}\right) g\left(\mathbf{r}_{\alpha}-\mathbf{r}_{p}\right) \\
& \times f\left(\hat{k}_{r \alpha}, \hat{k}_{0}\right) g(R) \mathbf{E}_{0} .
\end{aligned}
$$

Depolarization effects (which will turn out to be a relatively small error in view of the fact that the receiving beam width is less than a milliradian so the intermediate scattering occurs close to the polar axis $z$ between the transmitter and the particle at $z=R$ ) will be ignored for the time being. This contribution is illustrated in Fig. 1 (which shows an exaggeratedly wide receiving-beam pattern for clarity). In Eq. (7) we replace the summation by a volume integration with a particle-size distribution factor $n_{4}(D)$, which is assumed to be independent of position. Under the assumption of particle incoherence, the associated power with this second-order process is

$$
\begin{aligned}
P_{\mathrm{bs}}^{(2)}= & \frac{1}{(4 \pi)^{6}} \int \mathrm{d} v_{\alpha} \int_{0}^{\infty} \mathrm{d} D n_{4}(D) \\
& \times \frac{\exp \left[-2 \gamma\left(l_{1}+l_{2}+R-R_{0}\right)\right]}{l_{3}{ }^{2} l_{2}{ }^{2} R^{2}} \\
& \times\left|f_{\text {Mie }}\left(\pi-\theta_{r \alpha}, D\right)\right|^{2}\left|f_{\text {Mie }}\left(\theta_{\alpha}, D\right)\right|^{2}\left|f_{\mathrm{bw}}\left(\theta_{0 \alpha}\right)\right|^{2}, \\
l_{1} \equiv & \left|\mathbf{r}_{1}-\mathbf{r}_{\alpha}\right|, \quad l_{2} \equiv\left|\mathbf{r}_{\alpha}-\mathbf{r}\right|, \quad l_{3} \equiv\left|\mathbf{r}_{\alpha}-\mathbf{r}_{p}\right|, \\
R_{0} \equiv & \left|\mathbf{r}-\mathbf{r}_{0}\right|, \quad R=\left|\mathbf{r}-\mathbf{r}_{p}\right| .
\end{aligned}
$$

The integrand has been multiplied by a factor $\mid f_{\mathrm{bw}}\left(\left.\theta_{0 \alpha}\right|^{2}\right.$ to account for the beam-width pattern of the receiving antenna. The following is a useful approximation for a lidar receiver with a half-power width of $\sim 0.0005$ rad:

$$
\mid f_{\text {bw }}\left(\left.\theta\right|^{2}=\exp \left(-2.77 \times 10^{6} \theta^{2}\right)\right.
$$

if $\theta$ is given in radians. Obviously Eqs. (8) are difficult to evaluate because at least two integrations are involved in $\mathrm{d} v_{\alpha}=2 \pi l_{\alpha}^{2} \mathrm{~d} l_{a} \sin \theta_{r a} \mathrm{~d} \theta_{r a}$. Because a typical size distribution is sharply peaked and can be 
modeled by a gamma distribution, e.g., as $n_{4}(D)=$ $6.863 \times 10^{-14} D^{6} \exp (-0.7 D) \mu \mathrm{m}^{-4}$ (if $D$ is in micrometers; the numerical coefficient is chosen to yield 600 particles per cubic centimeter), it follows that we might set $D=\langle D\rangle=10 \mu \mathrm{m}$, the average diameter, in the arguments of $f_{\text {Mie }}$ in Eqs. (8) to obtain

$$
\begin{aligned}
P_{\mathrm{bs}}^{(2)}= & \frac{2 \pi n_{3}}{(4 \pi)^{6}} \int_{0}^{\pi / 2} \mathrm{~d} \theta_{r a} \sin \theta_{r a} \\
& \times \int_{0}^{l_{f}} \mathrm{~d} l_{a} \frac{\exp \left[-2 \gamma R\left(l_{1}+l_{2}+R-R_{0}\right)\right]}{l_{3}^{2} R^{2}} \\
& \times\left|\bar{f}_{\text {Mie }}\left(\pi-\theta_{r \alpha}\right)\right|^{2}\left|\bar{f}_{\text {Mie }}\left(\theta_{\alpha}\right)\right|^{2}\left|f_{\text {bw }}\left(\theta_{0 \alpha}\right)\right|^{2},
\end{aligned}
$$

where $\bar{f}_{\text {Mie }}(\theta) \equiv f_{\text {Mie }}(\theta,\langle D\rangle), n_{3}$ is the particle density in reciprocal cubic meters, and $l_{f}=R_{0} / \cos \theta_{r a}$. On division by $P_{\mathrm{bs}}{ }^{(1)}$ and introduction of the new variables $\zeta=z / R$ and $\eta=\rho / R$, we obtain the ratio

$$
\begin{aligned}
\frac{P_{\mathrm{bs}}^{(2)}}{P_{\mathrm{bs}}^{(1)}}= & \frac{n_{3} R^{2}}{8 \pi} \int_{0}^{\pi / 2} \mathrm{~d} \theta_{r a} \sin \theta_{r a} \\
& \times \int_{0}^{\overline{4}} \mathrm{~d} l_{a} \frac{\exp \left[-2 \gamma R\left(\bar{l}_{1}+\bar{l}_{2}-1+\zeta_{0}\right)\right]}{\bar{l}_{3}^{2}} \\
& \times \frac{\left|\bar{f}_{\mathrm{Mie}}\left(\pi-\theta_{r \alpha}\right)\right|^{2}\left|\bar{f}_{\mathrm{Mie}}\left(\theta_{\alpha}\right)\right|^{2}\left|f_{\mathrm{bw}}\left(\theta_{0 \alpha}\right)\right|^{2}}{\left|f_{\mathrm{Mie}}(\pi, D)\right|^{2}},
\end{aligned}
$$

with $\bar{l}=l / R$ for all values of $l$ and $\zeta_{0}=R_{0} / R$. It is useful to replace the particle density by the attenuation coefficient $\gamma$. We do this by using

$$
\begin{gathered}
\gamma=n_{3} \frac{2 \pi}{k}\left\langle\operatorname{Im}\left[f_{\text {Mie }}(0, D)\right]\right\rangle, \\
\left\langle\operatorname{Im}\left[f_{\text {Mie }}(0, D)\right]\right\rangle=\frac{\int_{0}^{\infty} \mathrm{d} D n_{4}(D) \operatorname{Im}\left[f_{\text {Mie }}(0, D)\right]}{\int_{0}^{\infty} \mathrm{d} D n_{4}(D)} .
\end{gathered}
$$

The denominator in Eq. (12) is the density $n_{3}$ as an integral over size distribution. In that way we finally obtain

$$
\begin{aligned}
\frac{P_{\mathrm{bs}}^{(2)}}{P_{\mathrm{bs}}^{(1)}}= & \frac{k(2 \gamma R)}{32 \pi^{2}} \int_{0}^{\pi / 2} \mathrm{~d} \theta_{r a} \sin \theta_{r a} \\
& \times \int_{0}^{\bar{l}_{f}} \mathrm{~d} l_{a} \frac{\exp \left[-(2 \gamma R)\left(\bar{l}_{1}+\bar{l}_{2}-1+\zeta_{0}\right)\right]}{\bar{l}_{3}^{2}} \\
& \times \frac{\left|\bar{f}_{\mathrm{Mie}}\left(\pi-\theta_{r \alpha}\right)\right|^{2}\left|\bar{f}_{\mathrm{Mie}}\left(\theta_{\alpha}\right)\right|^{2}\left|f_{\mathrm{bw}}\left(\theta_{0 \alpha}\right)\right|^{2}}{\left\langle\operatorname{Im}\left[f_{\mathrm{Mie}}(0)\right]\right\rangle\left|f_{\mathrm{Mie}}(\pi, D)\right|^{2}}, \\
\sin \theta_{0 \alpha}= & \frac{\bar{l}_{2} \sin \theta_{r a}}{\bar{l}_{3}}, \quad \tan \theta_{\alpha}=\tan \left(\theta_{r a}+\theta_{0 \alpha}\right), \\
\bar{l}_{3}= & {\left[\left(\bar{l}_{2} \sin \theta_{r a}\right)^{2}+\left(1-\bar{l}_{2} \cos \theta_{r a}\right)^{2}\right]^{1 / 2}, } \\
\bar{l}_{1}= & \left(1-\frac{\zeta_{0}}{1-\bar{l}_{2} \cos \theta_{r a}}\right) \bar{l}_{3} .
\end{aligned}
$$

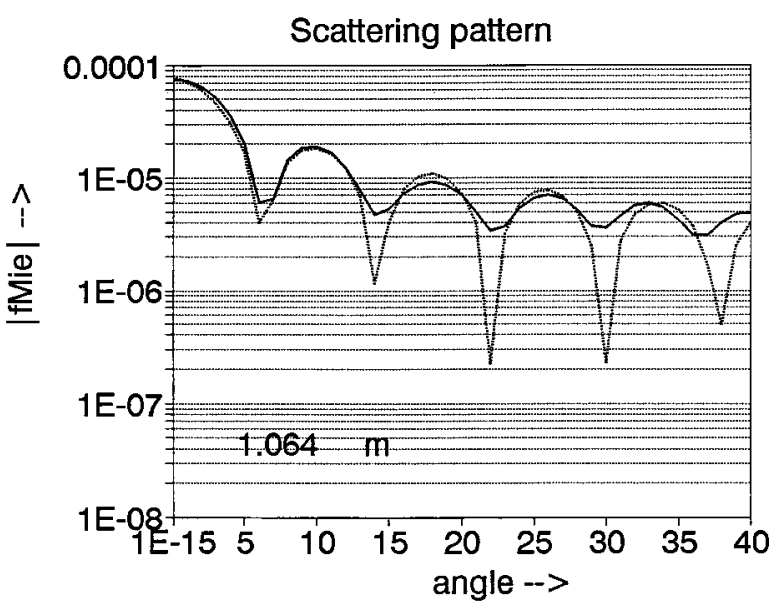

(a)

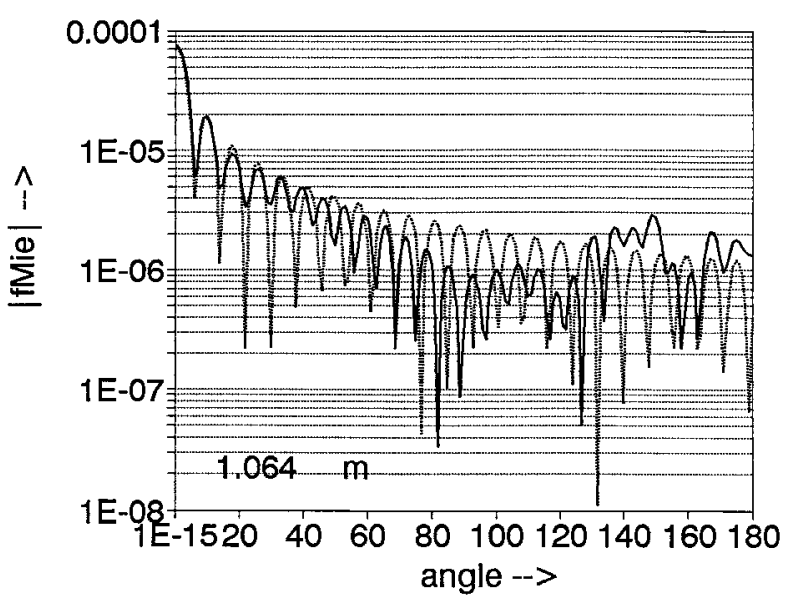

(b)

Fig. 2. Absolute value of the Mie-scattering coefficient as a function of scattering angle at $\lambda=1.064 \mu \mathrm{m}$ : (a) small angles, (b) all angles.

To obtain Eqs. (13) we used the monodisperse particle-distribution relationship for the attenuation coefficient, which depends only on $\operatorname{Im}\left[f\left(\hat{k}_{0}, \hat{k}_{0}\right)\right] \equiv$ $\operatorname{Im}\left[f_{\text {Mie }}(0,\langle D\rangle)\right]$ :

$$
\gamma=\frac{2 \pi}{k} n_{3} \operatorname{Im}\left[f_{\text {Mie }}(0)\right]
$$

to replace $n_{3}$ implied in Eq. (8) by $\gamma$. Equations (13) are the basis for numerical calculations discussed below.

\section{Numerical Calculations}

A basic input to Eqs. (13) is evaluation of the Mie coefficients $\operatorname{Re}\left[f_{\text {Mie }}(\theta,\langle D\rangle)\right]$ and $\operatorname{Im}\left[f_{\text {Mie }}(\theta,\langle D\rangle)\right]$ at the wavelengths of interest, in this case 1.064 and 0.532 $\mu \mathrm{m}$. At $10^{\circ} \mathrm{C}$, the refractive index for water ${ }^{22}$ at this wavelength is $n \approx 1.328+1.3 E-06 i$. A FORTRAN program derived by Bohren and Huffman ${ }^{1}$ was used to produce an angular pattern of the magnitude $\left|f_{\text {Mie }}(\theta,\langle D\rangle)\right|$ of the Mie coefficients in Figs. 2 and 3. Here, $\langle D\rangle$ has been chosen to be $10 \mu \mathrm{m}$. Careful study of the integrand in Eqs. (13) reveals that it is sizable only for not-large angles when the lidar is on 


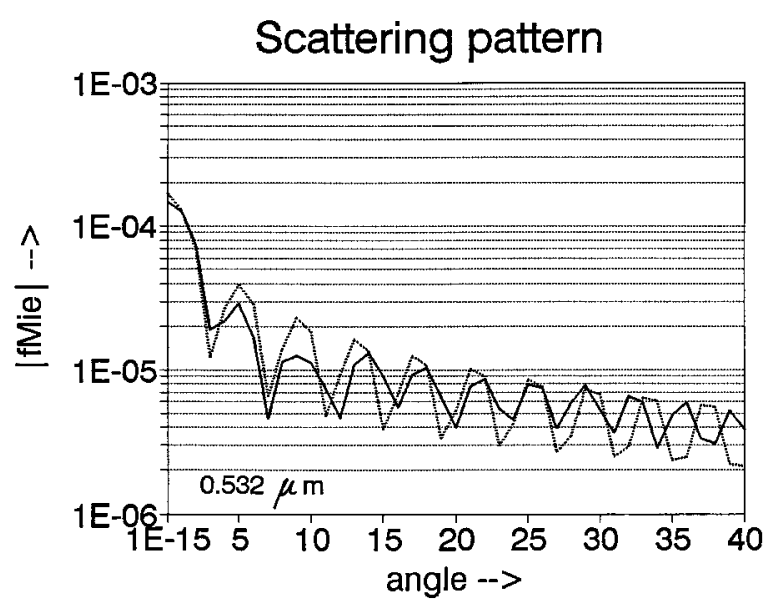

(a)

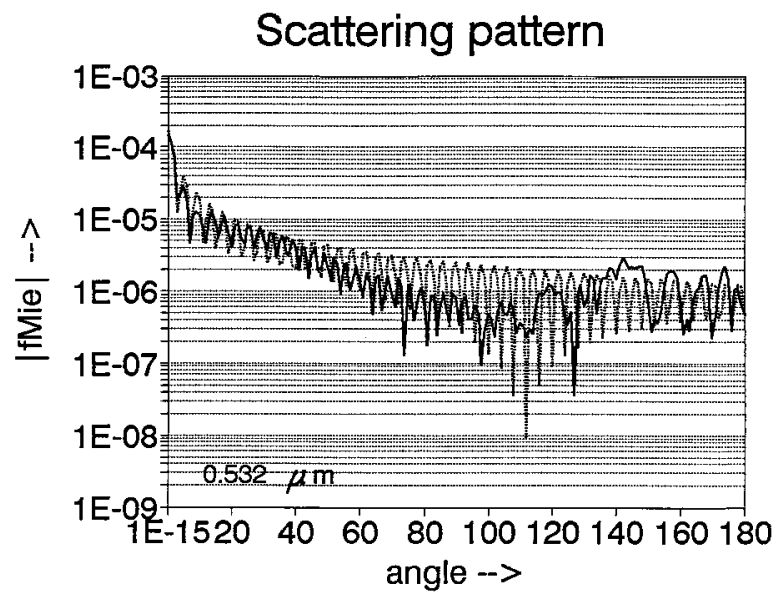

(b)

Fig. 3. Absolute value of the Mie-scattering coefficient as a function of scattering angle at $\lambda=0.532 \mu \mathrm{m}$ : (a) small angles, (b) all angles.

the ground and for angles close to $\pi / 2$ for a satellitebased receiver. This is due largely to the narrowness of the receiving beam width [see Eq. (9)]. A reasonable approximation at $\lambda=1.064 \mu \mathrm{m}$ (see Fig. 2 ), for $0<\left(\theta\right.$ in degrees) $<30^{\circ}$ (and for angles close to $\left.180^{\circ}\right)$, is

$$
\left|\bar{f}_{\text {Mie }}(\theta)\right| \approx 8.5 \times 10^{-5} \frac{|\sin (0.4 \theta+0.6)|}{|0.4 \theta+0.6|} .
$$

The approximation is shown as dotted curves in Fig. 2. The receiving beam-width factor is given in Eq. (9). The value of $\left\langle\operatorname{Im}\left[f_{\text {Mie }}(0)\right]\right\rangle$ can be approximated by $9.23 \times 10^{-5} \mathrm{~m}$, and that of $\left|f_{\mathrm{Mie}}(\pi, D)\right|^{2}$ by $1.79 \times$ $10^{-12} \mathrm{~m}$, at this wavelength and refractive index. Figure 4 then depicts the ratio $P_{\mathrm{bs}}^{(2)} / P_{\mathrm{bs}}^{(1)}$ as a function of attenuation $8.686 \gamma R$ at the above-mentioned wavelength. The dashed curves are the result of using a smoothed polynomial approximation instead of approximation (15):

$$
\begin{aligned}
\ln \left|\bar{f}_{\mathrm{Mie}}(\theta)\right| \approx & -10.2-0.0878 \theta+0.000618 \theta^{2} \\
& -1.282 \times 10^{-6} \theta^{3} .
\end{aligned}
$$

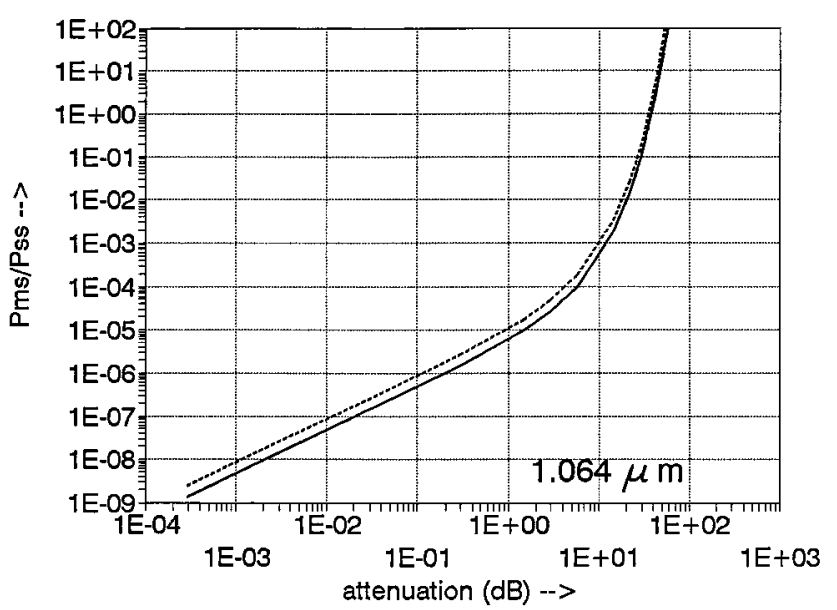

Fig. 4. Ratio of double-scattering to single-scattering power as a function of attenuation $8.686 \gamma\left(R-R_{0}\right)$ at $\lambda=1.064 \mu \mathrm{m}$ : ground observation.

It confirms that approximation (15) is reasonable over all angles. Figure 4 indicates, for this geometry and wavelength, that multiple scattering becomes appreciable at a one-way attenuation of $50 \mathrm{~dB}$. Now consider the following results for $\lambda=0.532 \mu \mathrm{m}$. The scattering pattern for $\left|f_{\mathrm{Mie}}(\theta,\langle D\rangle)\right|$ is shown in Fig. 3. A numerical fit similar to approximation (15), shown as dashed curves, is

$$
\left|\bar{f}_{\mathrm{Mie}}(\theta)\right| \approx 1.8 \times 10^{-4} \frac{|\sin (0.78 \theta+0.6)|}{|0.78 \theta+0.6|},
$$

and a smoothed polynomial approximation (yielding little difference in Fig. 5) is

$$
\begin{aligned}
\ln \left|\bar{f}_{\mathrm{Mie}}(\theta)\right| \approx & -9.92-0.1076 \theta+0.0008756 \theta^{2} \\
& -2.257 \times 10^{-6} \theta^{3} .
\end{aligned}
$$

The calculation of Eq. (13) then yields the curves of Fig. 5, where the solid curve represents the fit with approximation (17) and the dashed curve the fit with

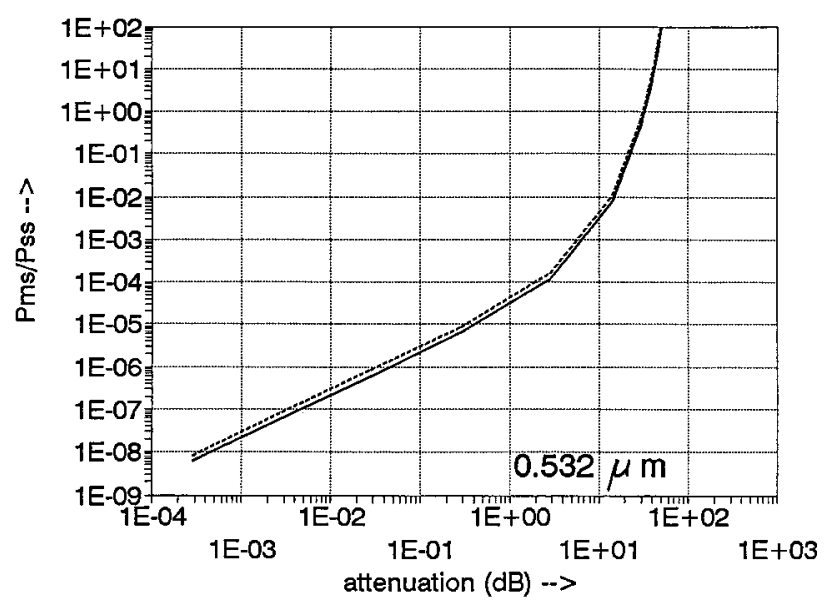

Fig. 5. Ratio of double-scattering to single-scattering power as a function of attenuation $8.686 \gamma\left(R-R_{0}\right)$ at $\lambda=0.532 \mu \mathrm{m}$ : ground observation. 


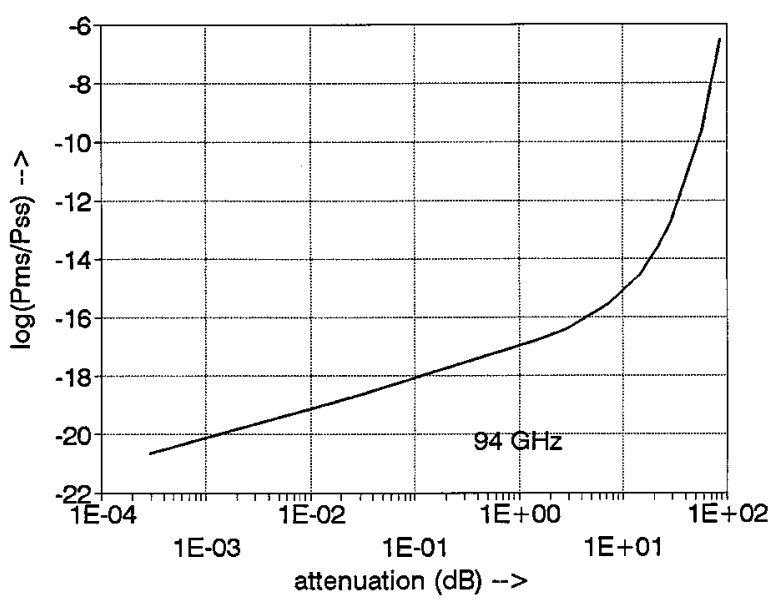

Fig. 6. Ratio of double-scattering to single-scattering power as a function of attenuation $8.686 \gamma\left(R-R_{0}\right)$ at $f=94 \mathrm{GHz}$ : ground observation.

approximation (18). The dominant process for both frequencies and small optical thickness (large $\gamma$ ) appears to be incoherent diffusion of electromagnetic power, which may be the reason that radiativetransfer calculations yield good results for this type of problem at optical frequencies for optically dense particulate media.

As a check, we have calculated a curve for a millimeter-wave frequency $(94 \mathrm{GHz})$ for which we can use the Rayleigh approximation, $\operatorname{Re}\left[f_{\text {Mie }}(\theta,\langle\mathrm{D}\rangle)\right]=$ $4.14 \times 10^{-10} \mathrm{~m}, \operatorname{Im}\left[f_{\mathrm{Mie}}(\theta,\langle D\rangle)\right]=8.26 \times 10^{-11} \mathrm{~m}$. The result of Eqs. (13) then is shown in Fig. 6. Clearly, the diffusion (incoherent multiple scattering) effect is negligible at this frequency.

In what follows, the calculations are repeated for satellite viewing of a $10-\mu \mathrm{m}$ particle that is embedded $0.5 \mathrm{~km}$ into a cloud that is $300 \mathrm{~km}$ below the satellite, so $R=300 \mathrm{~km}$ and $R_{0}=299.5 \mathrm{~km}$ (but point $\mathbf{r}_{p}$ now lies high above the layer, whereas $\mathbf{r}_{0}$ is at the lower boundary). The results at two optical wavelengths are shown in Figs. 7 and 8 . The ratios are larger (at

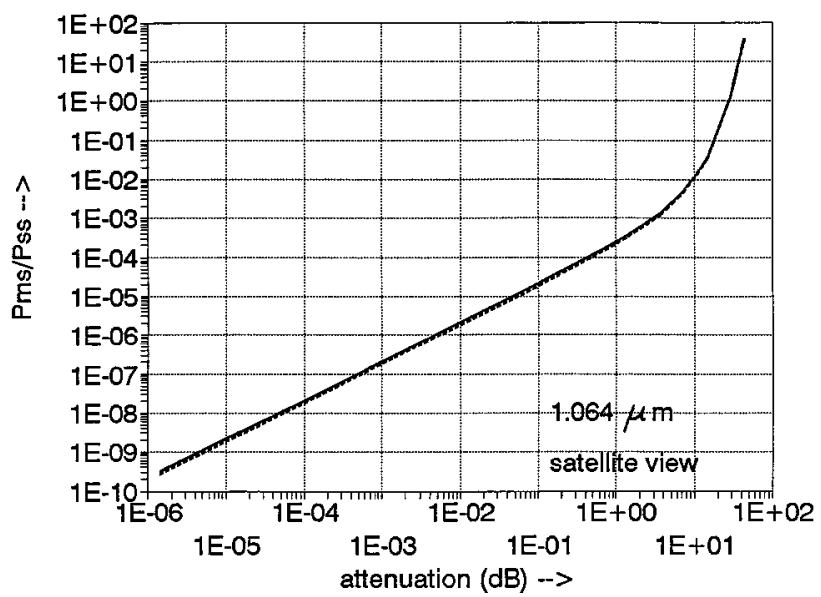

Fig. 7. Ratio of double-scattering to single-scattering power as a function of attenuation $8.686 \gamma\left(R-R_{0}\right)$ at $\lambda=1.064 \mu \mathrm{m}$ : satellite observation.

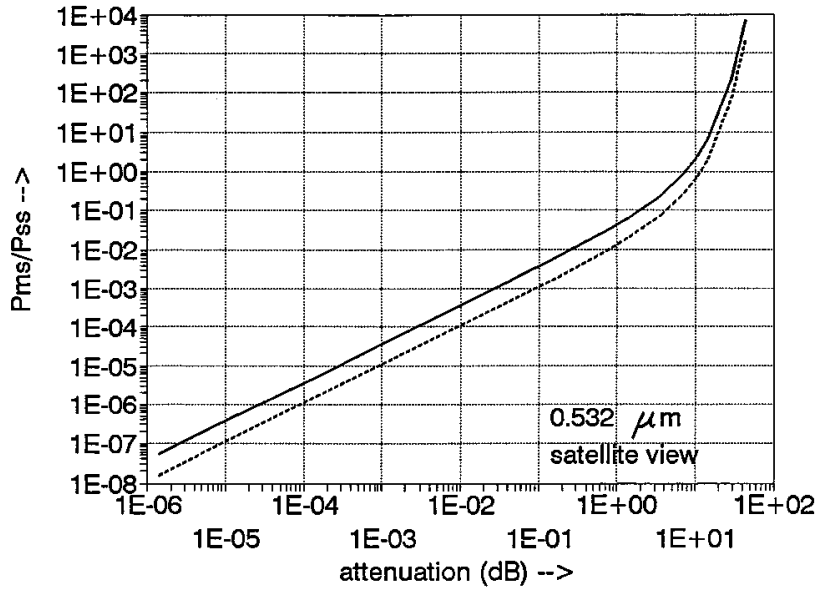

Fig. 8. Ratio of double-scattering to single-scattering power as a function of attenuation $8.686 \gamma\left(R-R_{0}\right)$ at $\lambda=0.532 \mu \mathrm{m}$ : satellite observation.

given $2 \gamma R$ ) than they are in the ground-viewing case. The main reason for this seems to be the wide intersection of the reception cone with a $0.5-\mathrm{km}$ layer at $300-\mathrm{km}$ distance, which gives a much larger region for secondary scatterings in a horizontal direction.

\section{Depolarization (Vectorial) Effects}

To account for the nonscalar quantities in Eq. (7) we need to know that a single spherical particle scatters a field $\mathbf{E}_{0}=E_{0 x} \hat{x}+E_{0 y} \hat{y}$ in the far field into two new orthogonal components ${ }^{1}$ :

$$
\left(\begin{array}{c}
E_{1 \theta} \\
E_{1 \varphi}
\end{array}\right)=\left[\begin{array}{cc}
f(\theta) \cos \theta & 0 \\
0 & f(\theta)
\end{array}\right]\left[\begin{array}{cc}
\cos \varphi & \sin \varphi \\
-\sin \varphi & \cos \varphi
\end{array}\right]\left(\begin{array}{l}
E_{0 x} \\
E_{0 y}
\end{array}\right),
$$

or

$$
\left(\begin{array}{c}
E_{1 \theta} \\
E_{1 \varphi}
\end{array}\right)=\left[\begin{array}{cc}
f_{1} \cos \theta & 0 \\
0 & f_{1}
\end{array}\right]\left(\begin{array}{l}
E_{0 \rho} \\
E_{0 \varphi}
\end{array}\right),
$$

given that the spherical-coordinate scattering angles are $(r, \theta, \varphi), \rho=r \sin \theta$, and $f_{1}=f(\theta)$. The geometry is depicted in Fig. 9, where two scatterings occur:

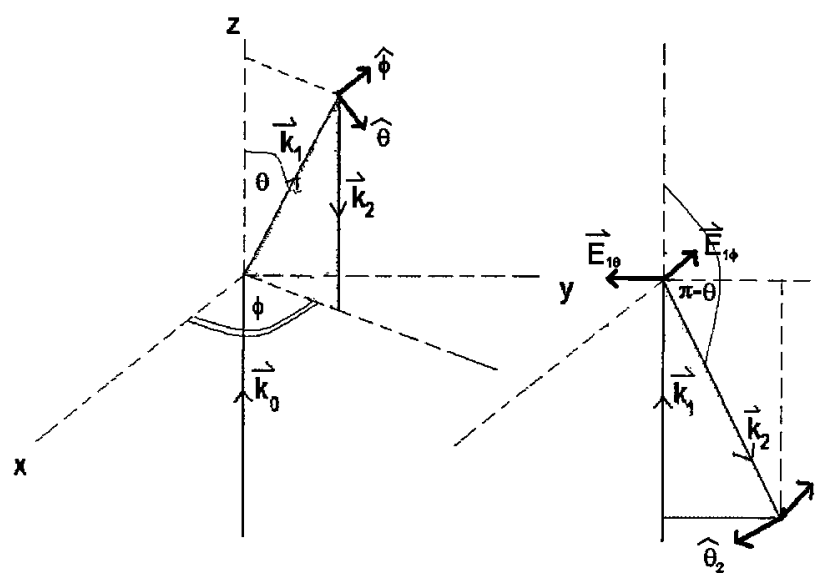

Fig. 9. Wave number and field vectors with relevant geometric scattering angles. 
from direction $\mathbf{k}_{0}$ to $\mathbf{k}_{1}$ and from direction $\mathbf{k}_{1}$ to $\mathbf{k}_{2}$. The field $\mathbf{E}_{0 \rho}$ is in the $\hat{\rho}=\hat{x} \cos \varphi+\hat{y} \sin \varphi$ direction. The scattered field amplitudes are $E_{1 \theta}$ in direction $\hat{\theta}$ and $E_{1 \varphi}$ in direction $\hat{\varphi}$. The connection between $E_{0 \rho}$ and $E_{0 \varphi}$ and the Cartesian coordinates $E_{0 x}$ and $E_{0 y}$ is obvious from Eq. (19), the notation of which is specifically for the first scattering.

For the second scattering, the following relationships can be inferred from Fig. 9: The new spherical angles after that scattering are $\hat{\theta}_{2}=-\hat{\rho}=-\hat{x} \cos \varphi-$ $\hat{y} \sin \varphi$ and $\hat{\varphi}_{2}=\hat{\varphi}=-\hat{x} \sin \varphi+\hat{y} \cos \varphi$. Moreover, $\theta_{2}=\pi-\theta$ and $\varphi_{2}=\pi / 2$. Thus $f_{2}=f(\pi-\theta)$. The second scattering is

$$
\begin{aligned}
\left(\begin{array}{l}
E_{2 \theta} \\
E_{2 \varphi}
\end{array}\right)= & {\left[\begin{array}{cc}
f_{2} \cos (\pi-\theta) & 0 \\
0 & f_{2}
\end{array}\right]\left[\begin{array}{cc}
\cos (\pi / 2) & \sin (\pi / 2) \\
-\sin (\pi / 2) & \cos (\pi / 2)
\end{array}\right] } \\
& \times\left(\begin{array}{l}
E_{1 x} \\
E_{1 y}
\end{array}\right) .
\end{aligned}
$$

Now use $E_{1 \varphi}=-E_{1 x}$ and $E_{1 \theta}=-E_{1 y}$ to convert Eq. (20a) into

$$
\left(\begin{array}{c}
E_{2 \theta} \\
E_{2 \varphi}
\end{array}\right)=\left[\begin{array}{cc}
f_{2} \cos \theta & 0 \\
0 & f_{2}
\end{array}\right]\left(\begin{array}{c}
E_{1 \theta} \\
E_{1 \varphi}
\end{array}\right)
$$

Use the transformation

$$
\left(\begin{array}{c}
E_{2 \theta} \\
E_{2 \varphi}
\end{array}\right)=\left[\begin{array}{cc}
-\cos \varphi & -\sin \varphi \\
-\sin \varphi & \cos \varphi
\end{array}\right]\left(\begin{array}{c}
E_{2 x} \\
E_{2 y}
\end{array}\right)
$$

to obtain for the cascaded two scatterings

$$
\begin{aligned}
& \left(\begin{array}{l}
E_{2 x} \\
E_{2 y}
\end{array}\right)=f_{1} f_{2}\left[\begin{array}{cc}
-\cos \varphi & -\sin \varphi \\
-\sin \varphi & \cos \varphi
\end{array}\right]\left[\begin{array}{cc}
\cos \theta & 0 \\
0 & 1
\end{array}\right] \\
& \times\left[\begin{array}{cc}
\cos \theta & 0 \\
0 & 1
\end{array}\right]\left[\begin{array}{cc}
-\cos \varphi & -\sin \varphi \\
-\sin \varphi & \cos \varphi
\end{array}\right]\left(\begin{array}{c}
E_{0 x} \\
E_{0 y}
\end{array}\right) \\
& =f_{1} f_{2}\left[\begin{array}{l}
\sin ^{2} \theta \cos ^{2} \varphi-\cos 2 \varphi \\
-1 / 2\left(1+\cos ^{2} \theta\right) \sin 2 \varphi
\end{array}\right. \\
& \left.\begin{array}{l}
-1 / 2\left(1+\cos ^{2} \theta\right) \sin 2 \varphi \\
\sin ^{2} \theta \cos ^{2} \varphi+\cos 2 \varphi
\end{array}\right]\left(\begin{array}{l}
E_{0 x} \\
E_{0 y}
\end{array}\right) .
\end{aligned}
$$

So, symbolically, we write Eq. (22) as

$$
\begin{aligned}
& E_{2 x}=f_{1} f_{2}\left[(a-b) E_{0 x}-c E_{0 y}\right], \\
& E_{2 y}=f_{1} f_{2}\left[-c E_{0 x}+(a+b) E_{0 y}\right],
\end{aligned}
$$

such that $a \equiv \sin ^{2} \theta \cos ^{2} \varphi, b \equiv \cos 2 \varphi$, and $c \equiv 1 / 2(1+$ $\left.\cos ^{2} \theta\right) \sin 2 \varphi$, as a result of which we obtain for the irradiance $\left|E_{2}\right|^{2}=\left|E_{2 x}\right|^{2}+\left|E_{2 y}\right|^{2}$ :

$$
\begin{aligned}
\left|E_{2}\right|^{2}= & \left|f_{1} f_{2}\right|^{2}\left\{\left(a^{2}+b^{2}+c^{2}\right)\left[\left|E_{0 x}\right|^{2}+\left|E_{0 y}\right|^{2}\right]\right. \\
& \left.-2 a b\left[\left|E_{0 x}\right|^{2}-\left|E_{0 y}\right|^{2}\right]-4 a c \operatorname{Re}\left[E_{0 x} E_{0 y}{ }^{*}\right]\right\} .
\end{aligned}
$$

The $2 a b$ and $4 a c$ terns vanish after integration of $\mathrm{d} \varphi$ from 0 to $2 \pi$. The remaining integrations, including that over $\sin \theta \mathrm{d} \theta$ from 0 to $\pi / 2$ (note that we integrate essentially over a half-space), finally yield

$$
\begin{aligned}
\int_{0}^{2 \pi} \mathrm{d} \varphi \int_{0}^{\pi / 2} \mathrm{~d} \theta \sin \theta\left|E_{2}\right|^{2}= & 1.6167 \pi\left|f_{1} f_{2}\right|^{2} \\
& \times\left(\left|E_{0 x}\right|^{2}+\left|E_{0 y}\right|^{2} .\right.
\end{aligned}
$$

Thus the net result of incorporating the polarization properties is that the ratio $\left|E_{2}\right|^{2} /\left|E_{0}\right|^{2}$ changes after angular integration in Eqs. (13) by a factor of $1.6167 \pi$ instead of by $2 \pi$, so Eqs. (13) requires a correction factor $1.6167 \pi / 2 \pi=0.8083$.

\section{Radiative-Transfer Formalism}

Equation (8) also can be derived by means of radiative-transport theory, ${ }^{11}$ and this is done briefly here. The basic integral equation for the specific intensity $I(l, \hat{\Omega})$ is

$$
\frac{\mathrm{d} I(\tau, \hat{\Omega})}{\mathrm{d} \tau}=-I(\tau, \hat{\Omega})+\frac{1}{\sigma_{t}} \int \mathrm{d}^{2} \Omega^{\prime}\left|f\left(\hat{\Omega}, \hat{\Omega}^{\prime}\right)\right|^{2} I\left(\tau, \Omega^{\prime}\right) .
$$

Here, $\tau=\int \mathrm{d} l n_{3}(l) \sigma_{t}(l)$ is the optical distance in the direction indicated by unit vector $\hat{\Omega}$, which in turn denotes the (straight) line along which the integral equation is being considered; $f\left(\hat{\Omega}, \hat{\Omega}^{\prime}\right)$ is the scattering amplitude for direction $\hat{\Omega}^{\prime}$ into direction $\hat{\Omega}$; and $\sigma_{t}$ is the extinction cross section, which is the sum of absorption cross section $\sigma_{a}$ and scattering cross section $\sigma_{s}=\int \mathrm{d}^{2} \Omega \mid f\left(\hat{\Omega},\left.\Omega^{\prime}\right|^{2}\right.$. As before, $n_{3}$ is the particle density. More generally, $\tau=\int \mathrm{d} l \int \mathrm{d}^{2} \Omega^{\prime} \int \mathrm{d} D$ $n_{4}(l, D)\left|f\left(\hat{\Omega}, \hat{\Omega}^{\prime}, D\right)\right|^{2}$ if we distinguish between different particle sizes. For a uniform monodisperse medium, one may set $\tau=\ln _{3} \sigma_{t}$, as a consequence of which Eq. (25) can be written more simply as

$$
\frac{\mathrm{d} I(l, \hat{\Omega})}{\mathrm{d} l}=-n_{3} \sigma_{t} I(l, \hat{\Omega})+n_{3} \int \mathrm{d}^{2} \Omega^{\prime}\left|f\left(\hat{\Omega}, \hat{\Omega}^{\prime}\right)\right|^{2} I\left(l, \Omega^{\prime}\right) .
$$

Application of the optical theorem to a unit volume of particles yields

$$
\begin{aligned}
n_{3} \sigma_{t} & =\frac{4 \pi}{k} n_{3} \operatorname{Im}[f(\hat{\Omega}, \hat{\Omega})] \\
& =\frac{4 \pi}{k} \int \mathrm{d} D n_{4}(D) \operatorname{Im}[f(\Omega, \Omega, \hat{D})] \equiv 2 \gamma .
\end{aligned}
$$

Here $\gamma$ is the extinction coefficient (in nepers per meter) for the medium, and consequently

$$
\frac{\mathrm{d} I(l, \hat{\Omega})}{\mathrm{d} l}=-2 \gamma I(l, \hat{\Omega})+n_{3} \int \mathrm{d}^{2} \Omega^{\prime}\left|f\left(\hat{\Omega}, \hat{\Omega}^{\prime}\right)\right|^{2} I\left(l, \Omega^{\prime}\right) .
$$




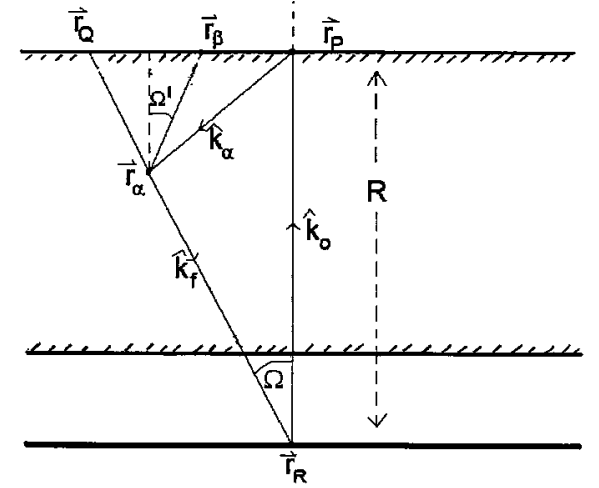

Fig. 10. Geometry relevant to the radiative-transfer calculation.

This can serve as an adequate starting point. This integral equation, with the aid of an integrating factor and a slight change of notation, becomes

$$
\begin{aligned}
I\left(\mathbf{r}_{P}, \hat{\Omega}\right)= & \exp \left(-2 \gamma l_{Q P}\right) I\left(\mathbf{r}_{Q}, \hat{\Omega}\right) \\
& +n_{3} \int_{Q}^{P} \mathrm{~d} l \exp \left[-2 \gamma\left(l_{P}-l\right)\right] \\
& \times \int \mathrm{d}^{2} \Omega^{\prime}\left|f\left(\hat{\Omega}, \hat{\Omega}^{\prime}\right)\right|^{2} I\left(l, \Omega^{\prime}\right) .
\end{aligned}
$$

Now we apply Eq. (30) several times to the sketch of Fig. 10. Let the received electric flux at point $\mathbf{r}_{R}$ be $S(R)=\int \mathrm{d}^{2} \Omega I\left(\mathbf{r}_{R}, \hat{\Omega}\right)\left|f_{\mathrm{bw}}(\Omega)\right|^{2}$, where we use the same receiving beam width factor as in Eq. (9). Apply Eq. (30) to this flux integral along the line $Q R$ to obtain two contributions, one from each part of Eq. (30). The first of these is

$$
S_{1}(R)=\int \mathrm{d}^{2} \Omega \exp \left(-2 \gamma l_{Q R}\right) I\left(\mathbf{r}_{Q}, \hat{\Omega}\right)\left|f_{\mathrm{bw}}(\Omega)\right|^{2} .
$$

It represents the contribution of the reduced incident intensity (Ref. 10, p. 158). However, $I\left(\mathbf{r}_{Q}, \hat{\Omega}\right)$ is nonzero, for the purpose of calculating two successive scatterings, only when point $Q$ is in the particle at point $P$ (as there is no zeroth-order source other than the particle at $P$ to produce radiation in direction $\hat{\Omega}$ ), at which point $\left|f_{\mathrm{bw}}(\Omega)\right|^{2} \approx\left|f_{\mathrm{bw}}(0)\right|^{2}=1$ and $\exp \left(-2 \gamma l_{Q R}\right) \approx \exp \left(-2 \gamma l_{P R}\right)=\exp (-2 \gamma R)$. Furthermore, in the immediate vicinity of the particle at $P$, the solid-angle increment can be written as $\mathrm{d}^{2} \Omega=$ $\mathrm{d} \mathscr{Y} / R^{2}$, where surface element $\mathrm{d} \mathscr{Y}$ is $\perp \hat{k}_{0}$. The direction $\hat{\Omega} \approx-\hat{k}_{0}$, and therefore

$$
S_{1}(R)=\frac{\exp (-2 \gamma R)}{R^{2}} \int \mathrm{d} \mathscr{I}\left(\mathbf{r}_{P},-\hat{k}_{0}\right)
$$

The surface integral $\int \mathrm{d} \mathscr{Y}\left(\mathbf{r}_{P},-\hat{k}_{0}\right)$, which extends only over the surface area of the particle subtended perpendicular to the direction $\hat{k}_{0}$, must be the power scattered per unit solid angle in direction $-\hat{k}_{0}$ and hence must equal $\left|f\left(-\hat{k}_{0}, \hat{k}_{0}\right)\right|^{2} S_{0}(P)$, where $S_{0}(P)$ is the incident power flux: $\mathbf{S}_{0}(P)=S_{0}(P) \hat{k}_{0}$. Thus

$$
S_{1}(R)=\frac{\exp (-2 \gamma R)}{R^{2}}\left|f\left(-\hat{k}_{0}, \hat{k}_{0}\right)\right|^{2} S_{0}(P)
$$

Now return to the received flux at $\mathbf{r}_{R}: \quad S(R)=\int \mathrm{d}^{2} \Omega$ $I\left(\mathbf{r}_{R}, \hat{\Omega}\right)\left|f_{\mathrm{bw}}(\Omega)\right|^{2}$, and insert the second term of Eq. (30) to obtain

$$
\begin{aligned}
S_{2}(R)= & n_{3} \int \mathrm{d}^{2} \Omega\left|f_{\mathrm{bw}}(\Omega)\right|^{2} \int_{\alpha}^{P} \mathrm{~d} l_{\alpha} \\
& \times \exp \left[-2 \gamma\left(l_{R}-l \alpha\right)\right] \\
& \times \int \mathrm{d}^{2} \Omega^{\prime}\left|f\left(\hat{\Omega}, \hat{\Omega}^{\prime}\right)\right|^{2} I\left(\mathbf{r}_{\alpha}, \Omega^{\prime}\right) .
\end{aligned}
$$

The last factor in Eq. (34) is a specific intensity at $\mathbf{r}_{\alpha}$ in the direction $\hat{\Omega}^{\prime}$ for which a separate expression that resembles Eq. (30) can be written down:

$$
\begin{aligned}
I\left(\mathbf{r}_{\alpha}, \hat{\Omega}^{\prime}\right)= & \exp \left(-2 \gamma l_{\beta \alpha}\right) I\left(\mathbf{r}_{\beta}, \Omega^{\prime}\right)+n_{3} \\
& \times \int_{\gamma}^{\alpha} \mathrm{d} l \exp \left[-2 \gamma\left(l_{\alpha}-l\right)\right] \ldots
\end{aligned}
$$

and for which specific intensity we have not written down the full expression, as only the first term of Eq. (35) will be inserted into Eq. (34) to yield an expression for two scatterings. Referring again to Fig. 9, we note that $I\left(\mathbf{r}_{\beta}, \hat{\Omega}^{\prime}\right)$ is nonzero only when $\mathbf{r}_{\beta} \approx \mathbf{r}_{P}$, so

$$
\begin{aligned}
\int \mathrm{d}^{2} \Omega^{\prime}\left|f\left(\hat{\Omega}, \hat{\Omega}^{\prime}\right)\right|^{2} \exp \left(-2 \gamma l_{\beta \alpha}\right) I\left(\mathbf{r}_{\beta}, \Omega^{\prime}\right) \\
\quad \approx\left|f\left(\hat{k}_{f}, \hat{k}_{\alpha}\right)\right|^{2} \exp \left(-2 \gamma l_{P \alpha}\right) \int \mathrm{d}^{2} \Omega^{\prime} I\left(\mathbf{r}_{P}, \hat{\Omega}^{\prime}\right) \\
\quad \approx\left|f\left(\hat{k}_{f}, \hat{k}_{\alpha}\right)\right|^{2} \frac{\exp \left(-2 \gamma l_{P \alpha}\right)}{l_{P \alpha}{ }^{2}} \int \mathrm{d} \mathscr{I} I\left(\mathbf{r}_{P}, \hat{\Omega}^{\prime}\right),
\end{aligned}
$$

in which expressions $\mathrm{d} \mathscr{S} \perp \hat{k}_{\alpha}$ and $\hat{\Omega}^{\prime}$ hardly deviates from direction $\hat{k}_{\alpha}$. For reasons similar to those that transform Eq. (32) into Eq. (33), it follows that

$$
\int \mathrm{d} \varphi I\left(\mathbf{r}_{P}, \hat{\Omega}^{\prime}\right)=\left|f\left(\hat{k}_{\alpha}, \hat{k}_{0}\right)\right|^{2} S_{0}(P)
$$

and therefore that approximation (36) becomes

$$
\begin{aligned}
& \int \mathrm{d}^{2} \Omega^{\prime}\left|f\left(\hat{\Omega}, \hat{\Omega}^{\prime}\right)\right|^{2} \exp \left(-2 \gamma l_{\beta \alpha}\right) I\left(\mathbf{r}_{\beta}, \Omega^{\prime}\right) \\
& \quad \approx n_{3} \frac{\exp \left(-2 \gamma l_{P \alpha}\right)}{l_{P \alpha}{ }^{2}}\left|f\left(\hat{k}_{f}, \hat{k}_{\alpha}\right)\right|^{2}\left|f\left(\hat{k}_{\alpha}, \hat{k}_{0}\right)\right|^{2} S_{0}(P) .
\end{aligned}
$$


The net result of all this is that Eq. (34) becomes

$$
\begin{aligned}
S_{2}(R)= & n_{3} \int \mathrm{d}^{2} \Omega \int \mathrm{d} l_{\alpha} \frac{\exp \left[-2 \gamma\left(l_{R}-l_{\alpha}\right)-2 \gamma\left(l_{P \alpha}\right)\right]}{l_{P \alpha}{ }^{2}} \\
& \times\left|f_{\mathrm{bw}}(\Omega)\right|^{2}\left|f\left(\hat{k}_{f}, \hat{k}_{\alpha}\right)\right|^{2}\left|f\left(\hat{k}_{\alpha}, \hat{k}_{0}\right)\right|^{2} S_{0}(P) .
\end{aligned}
$$

The final step is a replacement of $\mathrm{d}^{2} \Omega$ by $\mathrm{d} \mathscr{Y}_{\alpha} / l_{\alpha R}{ }^{2}$, where $\mathrm{d} \mathscr{Y}_{\alpha} \perp \hat{\Omega}$, so we obtain $\mathrm{d} \mathscr{Y}_{\alpha} \mathrm{d} l_{\alpha}$, which is the volume element $\mathrm{d} v_{\alpha}$ at point $\mathbf{r}_{\alpha}$. The result is

$$
\begin{aligned}
S_{2}(R)= & n_{3} \int \mathrm{d} v_{\alpha} \frac{\exp \left[-2 \gamma\left(l_{\alpha R}+l_{P \alpha}\right)\right]}{l_{\alpha R}{ }^{2} l_{P \alpha}{ }^{2}} \\
& \times\left|f_{\mathrm{bw}}(\Omega)\right|^{2}\left|f\left(\hat{k}_{f}, \hat{k}_{\alpha}\right)\right|^{2}\left|f\left(\hat{k}_{\alpha}, \hat{k}_{0}\right)\right|^{2} S_{0}(P) .
\end{aligned}
$$

This expression is substantially the same as Eq. (8); there are some differences in notation [e.g., the notation does not show explicitly that $l_{\alpha R}$ is nonzero only in the medium, and $n_{3}$ can be replaced by an integration over particle diameter with the distribution $n_{4}(D)$ such that the Mie factors can be those for a polydisperse rather than a monodisperse set of particles]. Also, a factor $(4 \pi)^{-6}$ is missing in approximation (39) because Ishimaru's ${ }^{13}\left[f_{\text {Mie }}\right]$ differs from the present $f_{\mathrm{Mie}}$ by a factor of $4 \pi$. Therefore, radiative transfer theory leads to the same expressions [Eqs. (6) and (8)] for direct backscatter and backscatter with one indirect intermediate scattering.

\section{Comment}

The scattering amplitude $f_{\mathrm{Mie}}(\theta, \lambda, \varepsilon, D)$ is a function not only of angle and wavelength but also of permittivity (or refractive index) and diameter of the spherical particle. As a result, Eq. (8) needs to be calculated without replacing $D$ by an average value in the Mie coefficients. The expression then is more difficult to evaluate because the three Mie factors in the integral over diameter $D$ are functions of $\eta$ and $\zeta$, as indicated in Eqs. (13), so the numerator is essentially a triple integral. However, this expression still would lead directly to Eqs. (13) for a monodisperse collection because the particle-size distribution $n_{4}(\zeta, D)$ would be a Dirac delta function in the diameter of the particles. Also, the widely different small-angle and smoothpolynomial approximations in Figs. 2 and 3-which lead to small differences in Figs. 4, 5, 7, and 8-seem to affirm that the lobed details of the Mie coefficients are not crucial here. Finally, the assumption that $n_{4}(D)$ is independent of $z$ is probably reasonable. The calculations of this study therefore lead to a reasonable estimate of conditions under which diffusionlike processes (radiative-transfer calculations) are more suited to obtaining estimates of backscatter from cloud particles.

\section{References and Notes}

1. C. F. Bohren and D. R. Huffman, Absorption and Scattering of Light by Small Particles (Wiley-Interscience, New York, 1983), Chap. 3.
2. H. C. van de Hulst, Light Scattering by Small Particles (Wiley, New York, 1957), Chap. 4.

3. M. Born and E. Wolf, Principles of Optics, 3rd ed. (Pergamon, Oxford, 1965), Sec. 2.4.2.

4. The denominator of the Lorentz-Lorenz formula for the effective refractive index reduces to a factor of 3 .

5. A. Van Lammeren, H. Russchenberg, A. Apituley, H. Ten Brink, and A. Feijt, "CLARA: a data set to study sensor synergy," presented at the Workshop on Synergy of Radar and Lidar in Space, Geesthacht, Germany, 12-14 November 1997.

6. Yu. A. Kravtsov and L. A. Apresyan, "Radiative transfer: new aspects of the old theory," in Progress in Optics, E. Wolf, ed. (Elsevier, Amsterdam, 1996), Vol. 36, pp. 200-212.

7. A. Mannoni, C. Flesia, P. Bruscaglioni, and A. Ismaeli, "Multiple scattering from Chebyshev particles: Monte Carlo simulations for backscattering in lidar geometry," Appl. Opt. 36, 7151-7164 (1996).

8. F. Nicolas, L. R. Bissonnette, and P. H. Flamant, "Lidar effective multiple scattering coefficients in cirrus clouds," Appl. Opt. 36, 3458-3468 (1997).

9. L. S. Xu, G. T. Zhang, and L. B. Cheng, "Parameterization of the shortwave radiative properties of water clouds for use in GCMS," Theor. Appl. Climatol. 55, 211-219 (1996).

10. C. Flesia and A. V. Starkov, "Multiple scattering from clear atmosphere obscured by transparent crystal clouds in satellite borne lidar sensing," Appl. Opt. 35, 2637-2641 (1996).

11. P. Bruscaglioni, "On the contribution of double scattering to the lidar returns from clouds," Opt. Commun. 27, 9-12 (1978).

12. L. R. Bissonnette, "Multiple scattering of narrow lightbeams in aerosols," Appl. Phys. B. 60, 315-323 (1995).

13. P. Bruscaglioni, A. Ismaeli, and G. Zaccanti, "Monte-Carlo calculations of lidar returns: procedure and results," Appl. Phys. B. 60, 325-329 (1995).

14. C. Flesia and P. Schwendimann, "Analytical multiplescattering extension of the Mie theory: the lidar equation," Appl. Phys. B. 60, 331-334 (1995).

15. A. V. Starkov, M. Noormohammadian, and U. G. Oppel, "A stochastic model and a variance-reduction Monte-Carlo method for the calculation of light transport," Appl. Phys. B. 60, 335-340 (1995).

16. D. M. Winker and L. R. Poole, "Monte-Carlo calculations of cloud returns for ground-based and space-based radars," Appl. Phys. B. 60, 341-344 (1995).

17. E. P. Zege, I. L. Katsev, and I. N. Polonsky, "Analytical solution to lidar return signals from clouds with regard to multiple scattering," Appl. Phys. B. 60, 345-353 (1995).

18. L. R. Bissonnette, P. Bruscaglioni, A. Ismaeli, G. Zaccanti, A. Cohen, Y. Benayahu, M. Kleiman, S. Egert, C. Flesia, P. Schendimann, A. V. Starkov, M. Noormohammadian, U. G. Oppel, D. M. Winker, E. P. Zege, I. L. Katsev, and I. N. Polonsky, "Lidar multiple scattering from clouds," Appl. Phys. B. 60, 355-362 (1995).

19. A. Ishimaru, Wave Propagation and Scattering in Random Media (Academic, New York, 1978), Vol. 2, Chap. 14.

20. L. Tsang, J. A. Kong, and R. T. Shin, Theory of Microwave Remote Sensing (Wiley, New York, 1985), Chap. 6.

21. Ref. 19, Vol. I, Chaps. 7 and 8; see also Ref. 11, pp. 381-382.

22. P. S. Ray, "Broadband complex refractive index of ice and water," Appl. Opt. 2, 1836-1844 (1972). 